DRAFT VERSION JUNE 20, 2018

Preprint typeset using LTEX style emulateapj v. 08/22/09

\title{
EXCITATION OF SLOW-MODES IN NETWORK MAGNETIC ELEMENTS THROUGH MAGNETIC PUMPING
}

\author{
YOSHIAKI KATO \\ Institute of Space and Astronautical Science, Japan Aerospace Exploration Agency, 3-1-1 Yoshinodai, Chuo-ku, Sagamihara, Kanagawa 252-5210, Japan
}

OSKAR STEINER

Kiepenheuer-Institut für Sonnenphysik, Schöneckstrasse 6, D-79104 Freiburg, Germany

Matthias STEFFEN

Astrophysikalisches Institut Potsdam, An der Sternwarte 16, D-14482, Potsdam, Germany

YOSHINORI SUEMATSU

Hinode Science Center, National Astronomical Observatory of Japan, 2-21-1 Osawa, Mitaka, Tokyo 181-8588, Japan

Draft version June 20, 2018

\begin{abstract}
From radiation magnetohydrodynamic simulations of the solar atmosphere we find a new mechanism for the excitation of longitudinal slow modes within magnetic flux concentrations. We find that the convective downdrafts in the immediate surroundings of magnetic elements are responsible for the excitation of slow modes. The coupling between the external downdraft and the plasma motion internal to the flux concentration is mediated by the inertial forces of the downdraft that act on the magnetic flux concentration. These forces, in conjunction with the downward movement, pump the internal atmosphere in the downward direction, which entails a fast downdraft in the photospheric and chromospheric layers of the magnetic element. Subsequent to the transient pumping phase, the atmosphere rebounds, causing a slow mode traveling along the magnetic flux concentration in the upward direction. It develops into a shock wave in chromospheric heights, possibly capable of producing some kind of dynamic fibril. We propose an observational detection of this process.

Subject headings: Sun: photosphere — Sun: chromosphere — Sun: oscillations — Sun: surface magnetism — magnetohydrodynamics (MHD)
\end{abstract}

\section{INTRODUCTION}

Filtergrams or spectroheliograms in $\mathrm{Ca}$ II $\mathrm{H}$ and $\mathrm{K}$ reveal two main sources of Ca II emission: plages, which spatially coincide with the active regions, and the chromospheric network, which outlines the boundaries of the supergranular velocity field (Simon \& Leighton 1964). Both are also the location of small magnetic flux concentrations in the photosphere and dynamic fibrils in the chromosphere (Hansteen et al. 2006). The close relationship between Ca II emission and magnetic flux (Skumanich et al. 1975; Schrijver et al. 1989) suggests that the magnetic field plays a key role for the chromospheric emission. The source of this emission has remained elusive, but likely candidates are the dissipation of magnetohydrodynamic waves, or the direct dissipation of electric currents. With regard to the former process, numerous studies have been carried out based on the approximation of slender flux tubes (see, e.g., Hasan \& Ulmschneider 2004, and references therein). They have greatly expanded our understanding of the physics of tube modes, mode coupling, dependency on the excitation spectrum and the tube geometry, shock formation, etc.

Some of these models consider the generation of tube waves by turbulent motions in the convection zone, where an analytical treatment of turbulence based on the Lighthill-Stein theory of sound generation is used, e.g., in Musielak et al. (1989, 1995, 2000) and Musielak \& Ulmschneider (2001). Others consider the generation of transverse waves through

Electronic address: kato.yoshiaki@isas.jaxa.jp the impulse transmitted by granules to magnetic flux tubes, as, e.g., in Choudhuri et al. (1993a b), Hasan et al. (2000), and Cranmer \& van Ballegooijen (2005).

Wave generation and propagation in thick tubes were considered by Ziegler \& Ulmschneider (1997a,b) who found the development of a non-axisymmetric longitudinal body wave and energy leakage by acoustic radiation. Huang et al. (1999) investigated surface and body waves of two-dimensional magnetic slabs. More recently Rosenthal et al. (2002), Bogdan et al. (2003), Hasan et al. (2005), Hasan \& van Ballegooijen (2008), Khomenko et al. (2008), and Vigeesh et al. (2009) carried out numerical simulations of magneto-acoustic wave propagation for an environment resembling the network atmosphere including small-scale magnetic flux concentrations with internal structure. They found that refraction of waves and mode conversion may play an important role in the conversion of wave energy to heat in the network.

All the above mentioned models and simulations impose a given driving, which is either monochromatic or impulsive, or derived from a theoretical spectrum of turbulence, or from observations. The focus of the present investigation is the selfconsistent excitation of a thick magnetic flux concentration through the ambient convective motion and specific consequences of this excitation.

\section{NUMERICAL METHOD AND MODEL CHARACTERISTICS}

The simulations were carried out with the $\mathrm{CO}^{5} \mathrm{BOLD}$ code (Freytag et al. 2002). This code solves the coupled 


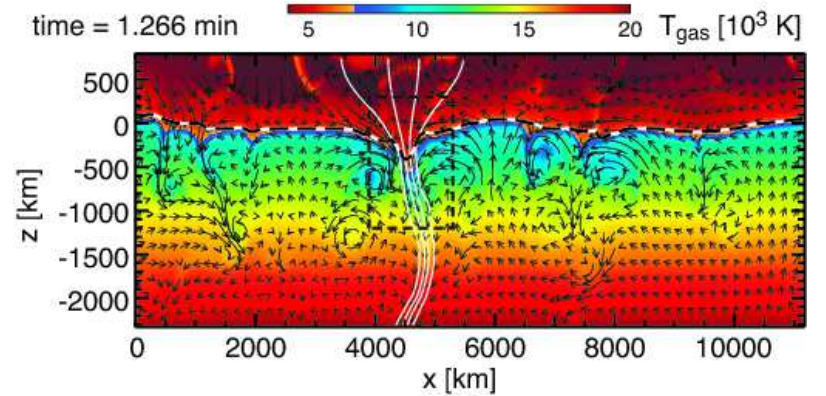

FIG. 1.- Snapshot of the simulation, showing the full computational domain. Color-scales show the gas temperature. The white solid curves are representative magnetic field lines, where the inner pair of field lines indicate the core region of the magnetic element and the outer pair of field lines the inner boundaries of the skin region. The black and white dashed curve shows the surface of optical depth unity, $\tau=1$. Arrows indicate the plasma velocity. The black dashed box indicates the size of the close-up of Figure 2

system of the equations of compressible magnetohydrodynamics in an external gravity field and non-local, frequencydependent radiative transfer in one, two, or three spatial dimensions. The main features of the code are described in Schaffenberger et al. (2005, 2006). The two-dimensional computational domain extends over a height range of $3160 \mathrm{~km}$ of which $780 \mathrm{~km}$ reach above the mean surface of optical depth unity. The horizontal extension is $11200 \mathrm{~km}$. The gridcell size in the horizontal direction is $28 \mathrm{~km}$, in the vertical direction it is $12 \mathrm{~km}$ in the upper part, continuously increasing to $30 \mathrm{~km}$ through the convection-zone. The lateral boundary conditions are periodic in all variables, whereas the lower boundary is open in the sense that the fluid can freely flow in and out of the computational domain under the condition of vanishing total mass flux. The specific entropy of the inflowing mass is fixed to a value previously determined so as to yield the correct solar radiative flux at the upper boundary. The upper boundary is transmitting, viz., $\mathrm{d} v_{x, z} / \mathrm{d} z=0$, while $\mathrm{d} \ln p_{\text {gas }} / \mathrm{d} z=-1 / H_{p}$ and $\mathrm{d} \ln \rho / \mathrm{d} z=-1 / H_{\rho}$ with $H_{p}$ and $H_{\rho}$ being the local gas pressure and density scale heights, respectively.

The simulation starts with a homogeneous, vertical, unipolar magnetic field of a flux density of $80 \mathrm{G}$ superposed on a previously computed, relaxed model of thermal convection. The magnetic field is constrained to have vanishing horizontal components at the top and bottom boundary but lines of force can freely move in the horizontal direction. At the beginning, the magnetic field quickly concentrates in the intergranular downdrafts of the convective flow. Subsequently, individual flux concentrations merge and after $\approx 100$ minutes, the magnetic field concentrates in a single magnetic 'flux sheet' with a strength of 2000 to $2600 \mathrm{G}$ at the optical depth unity within the flux concentration. This instant corresponds to the time $t=0$ in our investigation. Subsequently, the magnetic field remains concentrated in this single magnetic element for the remaining 86 minutes of simulation time.

This state, however, is not a stationary one-as a consequence of the interaction with the surrounding convective motion, the flux concentration moves laterally, gets distorted, and exhibits internal plasma flow. This interaction excites magneto-acoustic waves within the flux sheet, in particular longitudinal slow modes. In the following we point to a new mechanism of wave generation, which was not considered before.

\section{AMBIENT DOWNFLOWS AS A SOURCE OF SLOW MODES IN MAGNETIC ELEMENTS}

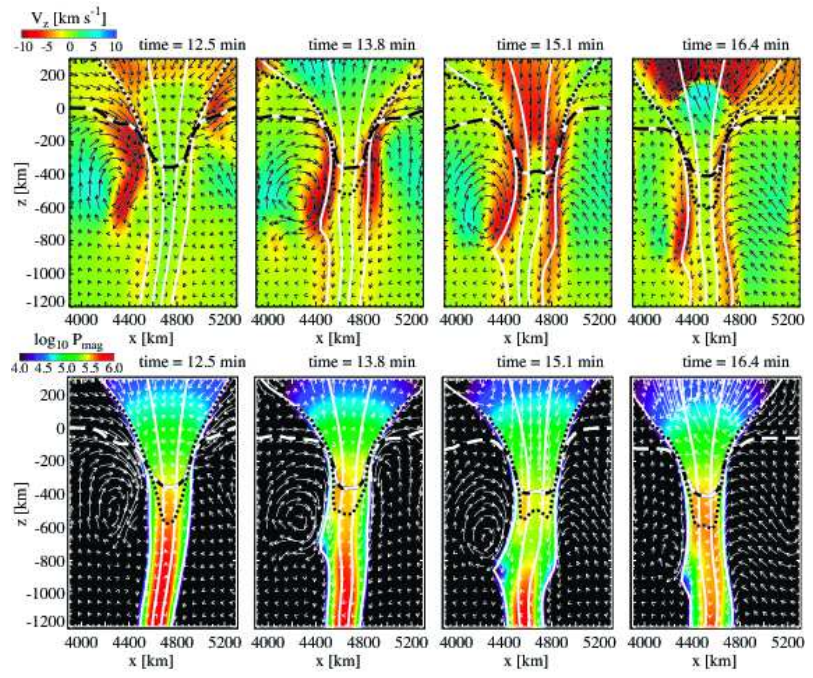

FIG. 2.- Time series of close-up views of the magnetic element, demonstrating the pumping by external downflows. Colors in the upper panels show the vertical velocity, colors in the lower panels the magnetic pressure. The white solid curves are representative field lines, where the inner pair of field lines indicate the core region and the outer pair the inner boundaries of the skin region. The dashed curves correspond to the surfaces of optical depth unity, $\tau=1$, the dotted curves to surfaces of equal magnetic and gas pressure, $\beta=1$, above which $\beta<1$. Negative velocities indicate downflows.

Figure 1 shows the complete computational domain with the magnetic flux concentration (white magnetic field lines) in the middle, the temperature field (color scale), the velocity field (arrows), and the continuum optical depth unity (dashed contour) for an arbitrary instant in time. The snapshot shows warm granular upwellings framed by narrower cool intergranular downdrafts in the convection zone and a few shock waves in the top part of the atmosphere. It also shows two narrow downflow channels in the close vicinity outside of the magnetic flux concentration around and below the surface of optical depth unity. These 'downflow jets' are a consequence of a baroclinic flow impinging on the magnetic flux concentration from the lateral directions, driven by the radiative cooling at the 'hot walls' of the flux concentration (Deinzer et al. 1984; Steiner et al. 1998). Observational evidences for downflows at the edges of small scale magnetic flux concentrations were reported by Rimmele (2004), Ishikawa et al. (2007), Langangen et al. (2007), and Rezaei et al. (2007).

In the present simulation, we observe that these downflows are far from stationary. They tend to be present most of the time but get transiently enhanced, weakened, or interrupted. Sometimes, the lateral inflow carries a preexisting regular intergranular downflow with it. It then merges with the preexisting downflow channel of the flux concentration, which results in a particularly strong downflow. Such advection of intergranular lanes to the downdrafts close to magnetic flux concentrations are reported to exist from observations of faculae by De Pontieu et al. (2006). In the following, we identify such transients as a source of magneto-acoustic waves within the magnetic flux concentration, in particular of longitudinal slow modes.

\subsection{The magnetic pumping process}

Figure 2 shows a close-up of a transient downflow and the ensuing excitation of a slow wave over a time period of $234 \mathrm{~s}$. The colors represent the vertical velocity in the top row and the magnetic pressure in the bottom row. The arrows indicate the flow speed, the vertically running white curves selected 
magnetic field lines of the magnetic flux concentration.

At the beginning of the time sequence, there exists on the left side of the flux concentration a vortical flow, which is directed towards the magnetic flux concentration near optical depth unity, turning into a fast downflow immediately adjacent to the flux concentration. A similar inflow and downflow exists on the right hand side of the flux concentration. In the course of time, these flow cells drift in the downward direction. The flows exert inertial forces on the magnetic flux sheet, which can be seen from the sequence of the magnetic pressure, $p_{\text {mag }}$.

Adjacent to where the flow impinges on the flux sheet, the magnetic pressure gets enhanced (e.g., at $z \approx-300 \mathrm{~km}$ at time 13.8 minutes and at $z \approx-500 \mathrm{~km}$ at time $15.1 \mathrm{~min}$ utes), while further downstream, where the flow speed is highest, and where the flow starts to detach from the flux-sheet boundary, the flux sheet expands and the magnetic pressure decreases. This situation leads to an inversion in $p_{\text {mag }}$ as a function of height, which moves in the downward direction together with the surrounding, gradually downward moving vortical flow cells. It presses material within the flux sheet in the downward direction. More illustratively expressed, the surrounding flow is (unidirectionally) kneading the flux sheet in the downward direction. This process entails a downflow within the magnetic flux concentration-while there exists a general upflow at time $t=12.5$ minutes, it turns into a downflow, first in the height range where the kneading is strongest (around $\tau=1$ ), later gradually comprising the upper atmospheric layers up to the top of the photosphere at time $t=15.1$ minutes. The mechanism by which convective flows in the surrounding of a magnetic flux tube may induce a unidirectional flow within the tube was earlier recognized and described by Parker (1974), who called this process 'turbulent pumping'. ${ }^{1}$ The principle is used in technical devices such as the peristaltic pump. Parker (1974) thought this process to be responsible for the intensification of magnetic flux concentrations. Here we find it to be responsible for the excitation of a magneto-acoustic slow mode.

\subsection{The generation of the slow mode}

At first, the kneading process transports all material in the downward direction, but this process is not efficient enough to prevent that some material can escape this trend and start to flow upwards in the center of the flux sheet, which is less directly subject to the surrounding flow. This upflow starts around $\tau=1$ at time $t=15.1$ minutes, while in the photosphere, plasma still flows at high speed in the downward direction. The high speed downflow collides with the upflowing material of higher density, which leads to an upwardly propagating wave. Since this takes place in the low $\beta$ regime, and since the wave is guided along the magnetic field, it is a slow, essentially acoustic wave. Further up it steepens into a shock front, visible at time $t=16.4$ minutes and $z \approx 200 \mathrm{~km}$.

Figure 3 shows two space-time diagrams of the event depicted in Figure 2. The left panel shows the vertical velocity (colors) and the gas pressure (contours) horizontally averaged over a $50 \mathrm{~km}$ thick skin region immediately outside of the magnetic flux sheet. The right panel shows the vertical velocity (colors) and the magnetic pressure (contours), horizontally averaged over a core region of the magnetic flux sheet, which

\footnotetext{
${ }^{1}$ Note that nowadays, this term commonly refers to the transport of magnetic fields by turbulence in stratified media, which is a different process. We therefore call this process 'magnetic pumping'.
}

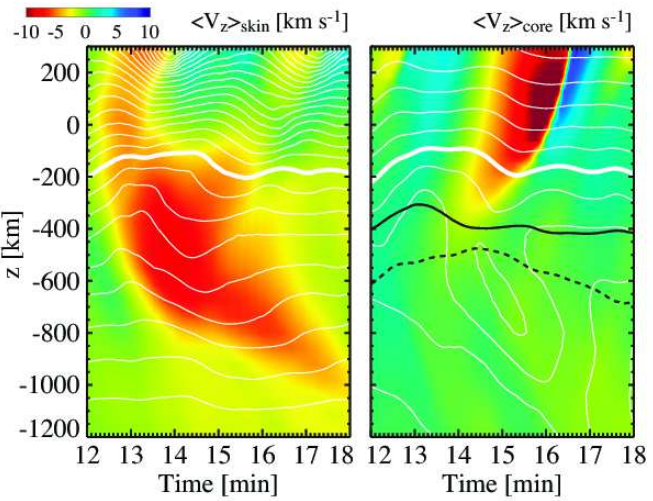

FIG. 3.- Space-time diagram of the close-up of Figure 2 between times $t=12$ minutes and $t=18$ minutes. Left: Skin region of the magnetic element. Colors refer to the vertical velocity, white contours to the logarithm of the gas pressure. Right: Core region of the magnetic element. Colors refer to the vertical velocity, white contours to the logarithm of the magnetic pressure. Negative velocities indicate downflows. Distances between white contours are equal in both panels. The gas pressure of the heavy white contour in the left panel is equal to the magnetic pressure of the heavy white contour in the right panel. Note that $\beta$ is not constant and very different for these two contours. The solid black curve indicates optical depth unity, the black dashed curve indicates the surfaces of equal gas pressure and magnetic pressure, $\langle\beta\rangle_{\text {core }}=1$. The strong downdraft in the skin region (red region) entails the downdraft in the magnetic element with subsequent shock formation starting at about $(t, z)=(16 \mathrm{~min} .,-50 \mathrm{~km})$.

encompasses one third of the total magnetic flux. The height $z=0$ corresponds to the mean optical depth unity outside of the flux sheet.

A downdraft in the flux-sheet surroundings (left panel) starts at a height of $z \approx 0 \mathrm{~km}$ and propagates downwards while strengthening: it also entails some replenishment from photospheric layers. The downdraft front is accompanied by an enhancement in gas pressure, which later turns into a gas pressure deficit as a consequence of Bernoulli's principle. Within the flux sheet (right panel), a downdraft ensues, first at time $t \approx 13.3$ minutes closely below the $\langle\tau\rangle_{\text {core }}=1$ level at height $z \approx-400 \mathrm{~km}$. This downdraft cannot be replenished from the lateral direction (unlike in the skin region) but is forced to flow along the magnetic field from higher layers. Consequently, a fast downflow ensues from $z \approx-300 \mathrm{~km}$ upwards. This downflow soon rebounds and turns into a upflow, developping into a shock front, visible from $(t, z)=$ $(16 \mathrm{~min} .,-50 \mathrm{~km})$ to $(t, z)=(16.6 \mathrm{~min} .,+300 \mathrm{~km})$. At $z=300 \mathrm{~km}$, the shock front moves with a speed of approximately $15 \mathrm{~km} \mathrm{~s}^{-1}$. The Alfvén speed at this height is $\approx 200 \mathrm{~km} \mathrm{~s}^{-1}$.

Figure 4 a) shows the space-time diagram of the vertical velocity for the full time period of 86 minutes. The colors above the height level of continuum optical depth unity, $\langle\tau\rangle_{\text {core }}=1$, refer to the vertical velocity in the core region of the flux concentration. Below $\langle\tau\rangle_{\text {core }}=1$, they refer to $v_{z}$ in the skin region. $z=0$ corresponds to the optical depth unity outside of the flux sheet, $\langle\tau\rangle_{\text {ext }}=1$. From this diagram it becomes evident that some of the most vigorous downflows in the photospheric layers of the flux sheet, e.g., at times $t=5.5,8.8$, $15.1,21.4,62.8,68.8$, and 77.5 minutes, are causally related to precursory downflows in the skin region of the flux sheet. The downflows in the skin region start at around optical depth unity. This is also visible in Figure 4(b), which shows the vertical velocity in the skin region to the left (blue) and to the right (red) of the flux sheet at a height of around $z=0$. The solid black curve gives the vertical velocity within the flux sheet at the same height. Strong downflows and subsequent 


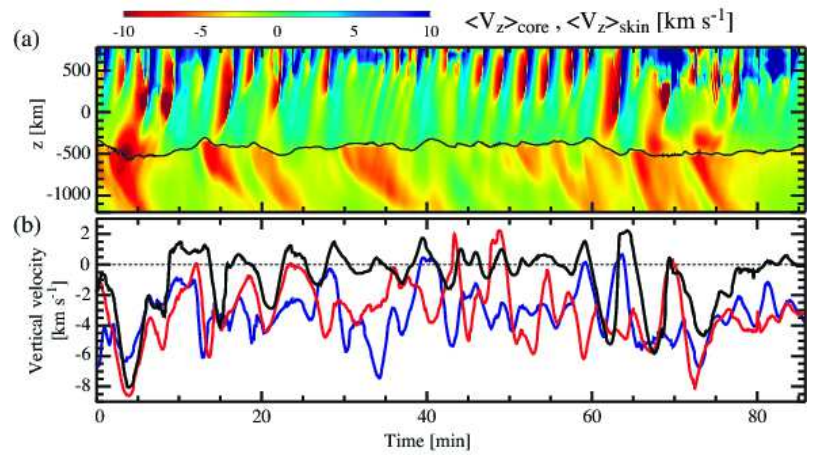

FIG. 4.- Top panel: Space-time diagram over the full time span of the simulation. The black solid curve corresponds to the $\langle\tau\rangle_{\text {core }}=1$ surface in the core of the flux sheet. Colors refer to the vertical velocity in the core region of the magnetic element above $\langle\tau\rangle_{\text {core }}=1$ and in the skin region of the magnetic element below $\langle\tau\rangle_{\text {core }}=1$. Bottom panel: Vertical velocity spatially averaged between $-500 \mathrm{~km} \leq z \leq 0 \mathrm{~km}$ as a function of time. The red curve refers to the skin region on the right hand side of the magnetic element, the blue curve to the skin region on the left hand side. The black curve refers to the core region of the magnetic element. Negative velocities indicate downflows.

shock formation in the photospheric layer of the magnetic flux concentration are often preceded by convective downflows in the close surroundings outside of the flux concentration.

From Figure 4(b) one can see that most of the time there exists a downdraft in the close surroundings of the magnetic flux concentration. However, strong downflow events occur sporadically only because they are triggered by the unsteady convective motion. Such sporadic events are capable of exciting a non-linear, longitudinal wave within the magnetic flux concentration as a consequence of the pumping effect. In the wake of this impulsive excitation, the atmosphere within the flux sheet oscillates at the acoustic cut-off period (Rae \& Roberts 1982; Hasan \& Kalkofen 1999). This is the reason for the predominantly periodic pattern in the upper part of Figure 4(a), despite of the sporadic, intermittent excitation by the ambient downflows.

\section{CONCLUSIONS AND DISCUSSIONS}

We have carried out a two-dimensional MHD simulation of a network magnetic element embedded in a nonstationary environment consisting of a $2.38 \mathrm{Mm}$ thick convection zone layer, and photospheric and chromospheric layers up to $0.78 \mathrm{Mm}$ above the optical depth $\tau=1$. In these simulations we found a new mechanism for the generation of longitudinal slow modes, which was not considered before. Sporadic strong downflows in the close surroundings of the magnetic element, 'pump' the plasma within the magnetic flux element in the downward direction by the action of inertial forces on the magnetic field-a process that was first described by Parker (1974) who referred to it as 'turbulent pumping'. As soon as the transient ambient downflow weakens, the pumping comes to a halt and the downflowing plasma from the photospheric and chromospheric layers of the magnetic element rebounds, which leads to a slow, upwardly propagating magneto-acoustic wave. It develops into a shock wave in the chromospheric layers of the magnetic element. In the wake of a 'pumping event', the flux-tube atmosphere tends to oscillate at the acoustic cutoff-period. We identify these pumping events as a crucial mechanism for the excitation of longitudinal slow modes in flux-tube atmospheres, the heating in network and plage areas, and possibly the development of some kind of dynamic fibrils.

A major shortcoming of the present investigation is the restriction to two spatial dimensions, which might favor the simultaneous presence of downflows on both sides of the flux sheet. At this point we do not yet know to what extent the pumping mechanism carries over to a three-dimensional environment. Also, we cannot exclude that viscous forces (stemming from the implemented subgrid turbulence model) may add to some extent to the pumping in our simulation. However, the viscosity effect is judged minor as there is a rather sharp discontinuity in velocity across the $\beta=1$ contour, as can be seen from Figure 2 .

For a future observational detection of the magnetic pumping mechanism, we propose to measure Doppler velocities within and in the close surroundings of magnetic elements at highest possible spatial resolution, and for different spectral lines, similar to Langangen et al. (2007) or Bellot Rubio et al. (2001). Different from these observations however, the measurements should preferably result in bi-dimensional Doppler maps, which would enable to keep track of the magnetic element. They should be supplemented with G-band and $\mathrm{Ca}$ II $\mathrm{H} / \mathrm{K}$ filtergrams for the identification of magnetic elements and the detection of the chromospheric response, respectively. In a time series of such measurements, we would expect a transient downdraft in the immediate surroundings of a magnetic element to entail a downflow in the photospheric layers within the magnetic element, followed by a fast upflow and a brightening in $\mathrm{Ca}$ II $\mathrm{H} / \mathrm{K}$ as a consequence of the shock wave.

O.S. gratefully acknowledges financial support and gracious hospitality during his visiting professorship at the National Astronomical Observatory of Japan (NAOJ), when part of the work reported herein was carried out. This work was supported in part by the JSPS fund \#R53 ("Institutional Program for Young Researcher Overseas Visits", FY20092011) allocated to NAOJ, part of which is managed by Hinode Science Center, NAOJ. The numerical simulations were carried out on the NEC SX-9 computer at JAXA Supercomputer Systems (JSS). We are grateful to R. Hammer for insightful discussions and to the referee for very helpful comments.

\section{REFERENCES}

Bellot Rubio, L. R., Rodríguez Hidalgo, I., Collados, M., Khomenko, E., \& Ruiz Cobo, B. 2001, ApJ, 560, 1010

Bogdan, T. J., et al. 2003, ApJ, 599, 626

Choudhuri, A. R., Auffret, H., \& Priest, E. R. 1993a, Sol. Phys., 143, 49

Choudhuri, A. R., Dikpati, M., \& Banerjee, D. 1993b, ApJ, 413, 811

Cranmer, S. R., \& van Ballegooijen, A. A. 2005, ApJS, 156, 265

De Pontieu, B., Carlsson, M., Stein, R., Rouppe van der Voort, L., Löfdahl,

M., van Noort, M., Nordlund, Å., \& Scharmer, G. 2006, ApJ, 646, 1405

Deinzer, W., Hensler, G., Schüssler, M., \& Weisshaar, E. 1984, A\&A, 139, 435
Freytag, B., Steffen, M., \& Dorch, B. 2002, Astron. Nachr., 323, 213

Hansteen, V. H., De Pontieu, B., Rouppe van der Voort, L., van Noort, M., \& Carlsson, M. 2006, ApJ, 647, L73

Hasan, S. S., \& Kalkofen, W. 1999, ApJ, 519, 899

Hasan, S. S., Kalkofen, W., \& van Ballegooijen, A. A. 2000, ApJ, 535, L67

Hasan, S. S., \& Ulmschneider, P. 2004, A\&A, 422, 1085

Hasan, S. S., \& van Ballegooijen, A. A. 2008, ApJ, 680, 1542

Hasan, S. S., van Ballegooijen, A. A., Kalkofen, W., \& Steiner, O. 2005,

ApJ, 631, 1270

Huang, P., Musielak, Z. E., \& Ulmschneider, P. 1999, A\&A, 342, 300 
Ishikawa, R., et al. 2007, A\&A, 472, 911

Khomenko, E., Collados, M., \& Felipe, T. 2008, Sol. Phys., 251, 589

Langangen, Ø., Carlsson, M., Rouppe van der Voort, L., \& Stein, R. F. 2007, ApJ, 655, 615

Musielak, Z. E., Rosner, R., Gail, H. P., \& Ulmschneider, P. 1995, ApJ, 448, 865

Musielak, Z. E., Rosner, R., \& Ulmschneider, P. 1989, ApJ, 337, 470

- 2000, ApJ, 541, 410

Musielak, Z. E., \& Ulmschneider, P. 2001, A\&A, 370, 541

Parker, E. N. 1974, ApJ, 189, 563

Rae, I. C., \& Roberts, B. 1982, ApJ, 256, 761

Rezaei, R., Steiner, O., Wedemeyer-Böhm, S., Schlichenmaier, R., Schmidt, W., \& Lites, B. W. 2007, A\&A, 476, L33

Rimmele, T. R. 2004, ApJ, 604, 906

Rosenthal, C. S., et al. 2002, ApJ, 564, 508

Schaffenberger, W., Wedemeyer-Böhm, S., Steiner, O., \& Freytag, B. 2005 , in ESA Special Publication, Vol. 596, Chromospheric and Corona

Magnetic Fields, ed. D. E. Innes, A. Lagg, \& S. A. Solanki (Noordwijk:

ESA)
Schaffenberger, W., Wedemeyer-Böhm, S., Steiner, O., \& Freytag, B. 2006, in ASP Conf. Ser., Vol. 354, Solar MHD Theory and Observations: A High Spatial Resolution Perspective, ed. J. Leibacher, R. F. Stein, \& H. Uitenbroek (San Francisco, CA: ASP), 345

Schrijver, C. J., Cote, J., Zwaan, C., \& Saar, S. H. 1989, ApJ, 337, 964

Simon, G. W., \& Leighton, R. B. 1964, ApJ, 140, 1120

Skumanich, A., Smythe, C., \& Frazier, E. N. 1975, ApJ, 200, 747

Steiner, O., Grossmann-Doerth, U., Knölker, M., \& Schüssler, M. 1998, ApJ, 495, 468

Vigeesh, G., Hasan, S. S., \& Steiner, O. 2009, A\&A, 508, 951

Ziegler, U., \& Ulmschneider, P. 1997a, A\&A, 324, 417

-. 1997b, A\&A, 327, 854 